Amaltea. Revista de Mitocrítica

ISSN-e: 1989-1709

http://dx.doi.org/10.5209/AMAL.51778

\title{
Ariane et la Clef d'or
}

Pierre Brunel ${ }^{1}$

Recibido: 31 de enero de 2016 / Aceptado: 3 de julio de 2016

Résumé. L'œuvre de Paul Dukas ne peut pas être réduite à son œuvre la plus célèbre, L'Apprenti sorcier. Ayant pratiqué aussi la critique, Dukas apparaît comme un auteur avec un style original et propre différencié de celui de Debussy. Dans Ariane et Barbe-Bleue, malveillamment considérée comme une simple réponse à Pelléas et Mélisande, sa préoccupation principale reste la transformation en musique du poème de Maeterlinck, d'en sortir la musique interne du langage écrit.

Palabras clave: musique, Paul Dukas, Ariane et Barbe-Bleue, Debussy, Maeterlinck.

\section{Ariadne and the Golden Key}

\begin{abstract}
Paul Dukas' work cannot be reduced to his most famous piece, L'Apprenti sorcier. Having also published in many journals, he stands out as an author with original and individual style different from Debussy's. In Arianne et Barbe-Bleue, maliciously considered as just a response to Pelléas et Mélisande, his biggest concern remains the transformation into music of Maeterlinck's poem, the way of bringing the internal music out of written language.
\end{abstract}

Keywords: music, Paul Dukas, Ariane et Barbe-Bleue, Debussy, Maeterlinck.

Cómo citar: Brunel, P. (2016) Ariane et la Clef d'or, en Amaltea. Revista de Mitocrítica 8, 17-21.

En 2015, l'éloge de Paul Dukas compositeur n'est plus à faire et nul ne le réduirait aujourd'hui à son œuvre la plus célèbre, L'Apprenti sorcier, ce " Scherzo d'après une ballade de Goethe », qui, tout comme au jour de sa création, le 18 mai 1897, continue d'être salué avec l'enthousiasme d'un vaste public de tous les âges. Le grand chef d'orchestre Bruno Walter, rendant hommage au compositeur alors récemment disparu dans le numéro spécial que lui consacrait La Revue musicale, en mai-juin1936, reconnaissait qu'il s'était acquis " une célébrité universelle » avec ce poème symphonique, mais regrettait qu'il ne fût, à cause de cette œuvre, « trop souvent considéré que comme un musicien descriptif». «En réalité », précisait-il, «c'est un musicien de pursang, comme le prouvent, entre autres œuvres, sa belle et importante Symphonie et le somptueux poème de La Péri. En outre, c'est un grand musicien dramatique qui, après Debussy, a créé un style tout à fait original, plein de poésie et de force. Ariane et Barbe-Bleue, que je considère comme une des plus puissantes manifestations de l'Ecole française, indique une

1 Université Paris IV, Sorbonne (Paris)

E-mail: pierre.brunel@ccfs-sorbonne.fr 
voie à l'avenir ». C'était à la fois tisser la relation entre Pelléas et Mélisande et Ariane et Barbe-Bleue et marquer que l'unique opéra de Paul Dukas n'est nullement, comme l'ont prétendu certains critiques malveillants, « une contrefaçon ratée de Pelléas », mais une œuvre ayant son originalité propre, "ce naturel de fond qui le différencie de Debussy », comme l'écrivait la grande pianiste Yvonne Lefébure dans le même numéro d'hommage de La Revue musicale.

La critique, Paul Dukas lui-même l'avait beaucoup pratiquée, ne publiant pas moins de quatre cents articles dans La Revue hebdomadaire et dans La Gazette des beaux-arts, ainsi que dans d'autres journaux et revues, entre 1892 et 1932. Je me garderai d'oublier La Revue musicale déjà citée, à laquelle il a donné non seulement des textes, mais des œuvres musicales, en 1920 La Plainte au loin du faune, un hommage à Debussy, et pour saluer cette fois le Prélude à l'après-midi d'un faune, en 1924 un Sonnet pour chant et piano, tiré du premier livre des Amours de Ronsard.

L'un de ses articles, en mai 1902, était consacré à Debussy et à Pelléas et Mélisande accueilli dans une atmosphère houleuse le jour de la répétition générale à l'Opéra-Comique, le 27 avril précédent. Un autre, cinq ans plus tard, à lui-même et à son Ariane et Barbe-Bleue, représentée pour la première fois dans la même salle, le 10 mai $1907^{2}$.

Ce que Paul Dukas écrivait dans son article sur Pelléas et Mélisande pourrait parfaitement être repris à propos de son Ariane et Barbe-Bleue. «Un fait capital, et sur lequel on a trop peu insisté », soulignait-il, "c'est le caractère tout particulier de la collaboration de M. Maeterlinck et de M. Debussy. Ce n'est pas le moins du monde de livret et de partition qu'il s'agit ici ; mais de la transformation musicale d'un drame conçu indépendamment d'une adaptation lyrique possible ».

Certes Dukas se serait bien gardé de porter sur ce qu'il présente comme une «moralité à la façon des contes de Perrault» le jugement sévère de Jean-Marie Andrieu dans le livre qu'il a consacré à Maurice Maeterlinck: "Privée de musique, cette féerie tomberait dans le gratuit et l'arbitraire $»^{3}$. Comme le poème dramatique publié pour la première fois par l'éditeur Lacomblez à Bruxelles en 1892, Ariane et Barbe-Bleue, "conte en trois actes », confié au même éditeur en 1907, était un texte qu'on peut qualifier de musical et contenant sa propre musique. Dukas le reconnaît à propos de Pelléas et Mélisande, "drame littéraire » qui, écritil dans son article de mai 1902, " côtoie sans cesse ces régions du sentiment où l'expression verbale aspire à se perdre dans l'expression sonore. Il est musical par

2 Ce texte a été publié par La Revue musicale dans le numéro de mai-juin 1936, repris dans le numéro de L'Avant-scène Opéra (149-150) consacré en 1992 à Ariane et Barbe-Bleue ainsi qu'au Château de Barbebleue de BelaBartok (retirage numérique en octobre 2013), p. 14-17. Il sera cité ici d'après le volume préfacé par Jean-Vincent Richard, Chroniques musicales sur deux siècles 1892-1932 de Paul Dukas, Stock Musique, 1980, p. 155-158, où l'on trouve aussi, parmi la cinquantaine d'articles retenus, celui de mai 1902 sur Pelléas et Mélisande, p. 147-150.

3 Editions universitaires, coll. Classiques du XXe siècle, 1962, p. 76. 
l'atmosphère mystérieuse où baignent ses parties même les plus fortement arrêtées et les mieux en lumière. Il est musical, aussi, par la richesse harmonieuse du langage, par son dialogue aux phrases de sens lointain, dont l'orchestre seul peut prolonger et répercuter les échos ».

L'atmosphère mystérieuse, Ariane l'évoque dès le début du Premier Acte quand, répondant à l'accusation que lance la Nourrice contre le maître du château de Barbe-Bleue - « il a tué cinq femmes »-, elle corrige :

Elles ne sont pas mortes. On en parlait là-bas comme d'un mystère étrange, dans le pays lointain où son amour sauvage et qui tremblait pourtant, est venu me chercher.

La richesse harmonieuse du langage, elle éclate un peu plus loin quand la nouvelle venue, éblouie par les «clairs diamants » qui ruissellent sous ses yeux à l'ouverture de la sixième porte, salue cette « immortelle rosée de lumière » et voit dans ces feux «comme un peuple d'esprits qui sème des étoiles », car " c'est la passion de la clarté qui a tout pénétré, ne se repose pas, et n'a plus rien à vaincre qu'elle-même ».

Il a suffi, pour ouvrir les six premières portes, des six clefs d'argent remises par le maître des lieux à sa future épouse, celles « qui ouvrent les trésors des parures nuptiales ». Et c'est grâce à la sixième de ces clefs «permises » qu'en effet Ariane a pu ouvrir la porte de la chambre aux diamants. Mais il y en a une septième, une «clef d'or», une clef qui est "interdite» et que pourtant elle a déjà décidé d'utiliser, car elle a immédiatement pressenti que c'était la clef du mystère. «C'est la seule qui importe », dit-elle. - Je jette les six autres et garde celle-ci ».

Cette clef d'or, elle est celle qui permet au compositeur, non pas d'ajouter de la musique au texte sur lequel il travaille, mais d'en extraire la musique qu'il contient. Rimbaud avait décrit l'une de ses expériences poétiques comme une « alchimie du verbe ». Pour la musique, Paul Dukas sait, après le chef-d'œuvre de Claude Debussy, qu'elle procède d'une alchimie du texte à partir de ce que ce texte contient. Il suffit d'en trouver la clef, qui n'est pas clef d'ut, clef de sol ou clef de fa, mais clef d'or.

Le précédent était Pelléas et Mélisande. "Voilà, je crois, ce que M. Debussy a voulu faire », écrivait-il en mai 1902. "Il a, selon moi, absolument et magnifiquement réussi à entourer de l'atmosphère qui lui convenait le drame de Maeterlinck. Il y a réussi sans que son originalité musicale, ni la richesse de ses dons [...] l'aient fait dévier d'une ligne du chemin qu'il s'était tracé. La nouveauté du résultat devait être un motif d'étonnement pour les juges les mieux exercés ; beaucoup d'entre eux crurent devoir rapporter au poète toute son efficacité en déclarant qu'il n'y avait point là de musique, alors qu'au contraire il n'y a là que de la musique; mais une musique si naturellement incorporée à l'action, si naturellement jaillie de la situation, du décor et du langage, une musique si rapprochée de la musique incluse sous les mots que, dans l'impression totale produite par cette sorte de transfusion sonore, il devient impossible de la dissocier 
du texte qu'elle pénètre ; au point qu'en dernier lieu elle peut aussi bien apparaître l'œuvre inconsciente du poète que le poème celle du musicien ».

Cela n'interdit évidemment pas d'envisager la partition en elle-même, que ce soit celle de Pelléas et Mélisande, comme le faisait Paul Dukas lui-même à la fin de son article de mai 1902, que ce soit celle d'Ariane et Barbe-Bleue. Et je suis tenté de reprendre, à propos de l'œuvre de 1907, les termes mêmes qu'utilisait Dukas à propos de Pelléas, à cette différence près que cinq ans ont passé, que Debussy a ouvert la voie et que la surprise des auditeurs, la malveillance de certains critiques sont dépassées. Nul n'irait dire maintenant « qu'il n'y [a] là ni rythme, ni mélodie, ni harmonie», ou encore «nulle trace de développement thématique ni même de thèmes musicalement intelligibles ». Nul ne réduirait le traitement instrumental à «un agréable et monotone bruissement d'orchestre ronronnant au hasard ». Ce qui est certain, c'est que ni dans Pelléas ni dans Ariane il n'y a de morceaux ni d'airs à effet. La musique, non divisée en morceaux séparés, peut et doit trouver en elle-même son équilibre, sans que le rythme, la mélodie, l'harmonie puissent être considérées comme indépendantes de ce tout que constituent le poème de Maeterlinck et l'œuvre musicale qui en est issue.

« Pour percevoir pleinement et sans effort la beauté de l'œuvre du musicien », écrivait encore Dukas à propos de Pelléas et Mélisande, « il suffit de la contempler non pas du côté de l'ombre, des techniciens et des tiges de plomb, mais du côté de la lumière dont l'éclaire le poème. De là tout $\mathrm{s}$ 'anime et prend vie, toutes les phases de l'œuvre apparaissent directement sur un fond commun d'émotion et d'humanité, chaque mesure s'affirme correspondante au décor qu'elle souligne, du plus sombre au plus vibrant de clarté, et aux sentiments qu'elle peut rendre, des plus tendres, des plus passionnés, aux plus terribles et aux plus mystérieux ».

C'est pourquoi, pour Ariane comme pour Paul Dukas lui-même, il fallait délaisser les clefs d'argent, ouvrant des ruissellements d'améthystes, de saphirs, de perles, d'émeraudes, de rubis, de diamants, au profit de la seule clef d'or. Dès son arrivée, la nouvelle épouse de Barbe-Bleue, désireuse d'en découvrir le secret, sait qu'il faut ouvrir la porte défendue avec cette clef secrète. Elle était présentée par Maeterlinck lui-même dans le texte comme la clef du chant. Le chant remplit la salle et se répand partout dès que cette septième porte s'entr'ouvre. Ce chant véritablement personnifié par un groupe chantant évoque la "porte close» sur laquelle « les cinq filles d'Orlamonde », ont trouvé sans l'utiliser « une clef d'or », craignant qu'elle ne soit la porte de la mort.

Mais c'est de cette clef d'or qu'Ariane attend le salut, comme Dukas en attend le miracle de la musique. Sans doute, et il le signale dans son article de 1910, Ariane s'est-elle arrêtée un instant aux diamants "parce qu'ils symbolisent la passion de la clarté 'qui a tout pénétré, ne se repose pas et n'a plus rien à vaincre qu'elle-même' et parce que leur éclat magnifique et solaire l'emporte sur celui des autres pierreries ». L'équivalent en serait une musique trop brillante, trop séduisante, contre laquelle le compositeur lui aussi est en garde, sachant que la vraie lumière n'est pas donnée, mais conquise sur l'ombre.

Ariane et ses nouvelles compagnes, les autres femmes de Barbe-bleue qui portent des noms chers à Maeterlinck, dont Sélysette et Mélisande elle-même, se 
trouvent au Deuxième Acte dans une grotte, comme il y avait une grotte dans Pelléas et Mélisande, et une grotte d'abord pleine d'ombre. Mais la clef d'or n'a pas seulement permis d'ouvrir la serrure de la dernière porte, elle est, bien plus que la lampe de la Nourrice, l'instrument qui permet de trouver peu à peu la lumière, cette lumière qui, à la fin de ce Deuxième Acte est faite « des milliers de rayons dansant au creux des vagues ». Sélysette invite ses pauvres sœurs à danser la ronde de la lumière, et en effet elles disparaissent en dansant dans la clarté et en chantant,

Les cinq Filles d'Orlamonde

(La fée noire est morte)

Les cinq Filles d'Orlamonde

Ont trouvé les portes !...

$\mathrm{Au}$ Troisième Acte la séparation peu à peu va intervenir. Non pas à cause de l'action, devenue plus dramatique, avec la capture de Barbe-Bleue par les Paysans, la protection dont les femmes l'entourent, leur regroupement autour de lui. Même Mélisande à la chevelure de lumière ne le quittera pas. Au contraire Ariane, même si son rôle a été déterminant dans l'acte de charité, choisit de partir comme si cette lumière était encore trop incertaine. «Le bonheur que je veux ne peut vivre dans l'ombre », affirmait-elle déjà devant Barbe-Bleue à l'Acte I.

« Ariane est partie rejoindre son ailleurs mythique, laissant les femmes libres de leur choix ", constatait Jean-François Boubobza dans le commentaire littéraire et musical qu'il a écrit pour le numéro de L'Avant-Scène Opéra consacré en 1992 à Ariane et Barbe-Bleue (p. 65). Mais il avait bien compris que c'était aussi un retour au commencement. Mieux, que c'était une exigence musicale pour Dukas luimême. Et il citait pour terminer, comme je le fais moi-même, non pas le texte publié par Dukas en 1910, mais un texte antérieur, datant de 1894, où le compositeur suggérait que «la musique plus qu'aucun autre art, plus vivement même que la poésie, donne un corps aux aspirations vers l'infini ».

Telle était bien, pour le compositeur d'Ariane et Barbe-Bleue, la clef d'or de sa musique. 